INPLASY

PROTOCOL

To cite: Qiu et al. Impact of SGLT2 inhibitors and GLP1 receptor agonists on respiratory infections: a metaanalysis. Inplasy protocol 202110092. doi:

10.37766/inplasy2021.1.0092

Received: 23 January 2021

Published: 23 January 2021

Corresponding author:

Mei Qiu

13798214835@sina.cn

Author Affiliation:

Shenzhen Longhua District

Central Hospital

Support: None.

Review Stage at time of this submission: Preliminary searches.

Conflicts of interest:

None.

\section{Impact of SGLT2 inhibitors and GLP1 receptor agonists on respiratory infections: a meta-analysis}

Qiu, M1; Ding, LL2; Zhou, HR³ .

Review question / Objective: Whether use of sodium-glucose transporter 2 (SGLT2) inhibitors or glucagon-like peptide 1 (GLP1) receptor agonists significantly affects the incidence of respiratory infections is unestablished.

Condition being studied: This meta-analysis will evaluate the impact of drug treatment with SGLT2 inhibitors or GLP1 receptor agonists on the incidence of various respiratory infections.

Information sources: We will systematically search Embase and PubMed using appropriate search strategies, for identifying relevant randomized controlled trials (RCTs).

INPLASY registration number: This protocol was registered with the International Platform of Registered Systematic Review and Meta-Analysis Protocols (INPLASY) on 23 January 2021 and was last updated on 23 January 2021 (registration number INPLASY202110092).

\section{INTRODUCTION}

Review question / Objective: Whether use of sodium-glucose transporter 2 (SGLT2) inhibitors or glucagon-like peptide 1 (GLP1) receptor agonists significantly affects the incidence of respiratory infections is unestablished.
Condition being studied: This metaanalysis will evaluate the impact of drug treatment with SGLT2 inhibitors or GLP1 receptor agonists on the incidence of various respiratory infections.

\section{METHODS}

Search strategy: It is being improved. 
Participant or population: Adults with type 2 diabetes (T2D), adults with heart failure (HF), and adults with chronic kidney disease (CKD).

Intervention: Any SGLT2 inhibitor or GLP1 receptor agonist. We will not consider the dosage of drugs as an effect modifier.

\section{Comparator: Placebo.}

Study designs to be included: Cardiovascular or renal outcome RCTs in which each study arm includes more than 500 participants.

Eligibility criteria: As shown in the above PICOS criteria.

Information sources: We will systematically search Embase and PubMed using appropriate search strategies, for identifying relevant randomized controlled trials (RCTs).

Main outcome(s): 1. Overall respiratory tract infection, including upper respiratory tract infection and lower respiratory tract infection. 2. Upper respiratory tract infection. 3. Influenza. 4. Sinusitis. 5. Laryngitis. 6. Lower respiratory tract infection. 7. Bronchitis. 8. Pneumonia.

Data management: The articles identified by the retrieval of two online databases will be assessed for relevance according to their titles and abstracts, and then those potentially eligible studies will be assessed for the final eligibility according to the inclusion and exclusion criteria. Two authors will then independently extract the pre-specified data from the included studies using a standardized Excel data extraction sheet. The pre-specified data to be extracted contain study type, type of underlying diseases, type of intervention, type of control, the number of the occurrence of the respiratory infections of interest. Any disagreements relevant with study selection and data extraction will be resolved through discussion with a third author. Treatment effects will be measured by pooled risk ratios (RRs) and $95 \%$ confidence intervals (Cls) derived from meta-analysis of the dichotomous data as reported in original studies.

Quality assessment / Risk of bias analysis: Two authors will use the Cochrane risk of bias assessment tool to independently assess the quality of included RCTs. Any disagreements related to quality assessment will be resolved through discussion with a third author.

Strategy of data synthesis: We will use the study-level binary data (i.e., the number of events in the intervention group, the number of patients in the intervention group, the number of events in the control group, and the number of patients in the control group) to perform meta-analysis. Meta-analysis will be conducted respectively based on trials of SGLT2 inhibitors and those of GLP1 receptor agonists. Effect size will be presented as risk ratio (RR) and $95 \%$ confidence interval (CI). I2 statistic will be calculated to measure statistical heterogeneity. If $12>$ $50 \%$, meta-analysis will be done using the random-effects model. Otherwise, the fixed-effects model will be used. Cochran's $Q$ test will be used to test for treatment-bysubgroup interactions. Funnel plots and Egger tests will be done to assess the publication bias. All statistical analyses will be completed in the Stata/MP software (version 16.0).

Subgroup analysis: Subgroup analysis on all the endpoints of interest will be conducted according to type of underlying diseases.

Sensitivity analysis: Not preplanned.

Country(ies) involved: China.

Keywords: SGLT2 inhibitors, GLP1 receptor agonists, respiratory infections, pneumonia, bronchitis.

Contributions of each author:

Author 1 - Mei Qiu.

Author 2 - Liang-Liang Ding.

Author 3 - Hai-Rong Zhou. 